\title{
Singularities in the paramagnetism of two-dimensional nearly magnetic itinerant-fermion systems at very low temperature: Application to degenerate two-dimensional liquid- ${ }^{3} \mathrm{He}$ films
}

\author{
A. Theumann* and M. T. Béal-Monod \\ Laboratoire de Physique des Solides associé au Centre National de la Recherche Scientifique, \\ Université de Paris-Sud, F-91405 Orsay, France
}

(Received 13 July 1983)

\begin{abstract}
It is shown that two-dimensional (2D) paramagnon problems exhibit strong algebraic singularities $\left|q^{2}-4 K_{F}^{2}\right|^{-\alpha}$, when some relevant momentum $q$ is close to twice the Fermi momentum $K_{F}$. As all $q$ values from 0 to $2 K_{F}$ are equally relevant for the magnetic instability in $2 \mathrm{D}$, the above singularities play a key role. By contrast, they are irrelevant in 3D. It is explicitly shown that standard methods to calculate the uniform static susceptibility fail in 2D due to these singular terms. The origin of the singularities are multitail ring diagrams, closed fermion loops with "tails" attached to them. These diagrams are analyzed in detail by generalizing to $d$ dimensions and dynamic tails, the method of Brovman and Kagan, developed in another context. The subsequent effects in the Ginzburg-Landau-Wilson Lagrangian describing interacting paramagnons are dramatic and render such an expansion questionable. Moreover, the nature of the magnetic instability (ferromagnetic or antiferromagnetic type), which is not well defined in mean-field theory, still remains unsolved in the presence of paramagnons since standard methods to renormalize the response functions with fluctuations fail to apply in the 2D problem. Any naive transposition from 3D to 2D of the Landau Fermi-liquid theory to compute, for instance, properties of liquid- ${ }^{3} \mathrm{He}$ films are suspected to be premature-if not erroneous-at this stage.
\end{abstract}

\section{INTRODUCTION}

Three-dimensional (3D) nearly magnetic Fermi liquids, with strong spin interactions, have been extensively studied in the past, ${ }^{1}$ both experimentally and theoretically, in particular using a somewhat simplified version of the Landau theory, ${ }^{2}$ the paramagnon model. ${ }^{3}$ This model corresponds to a one-parameter theory, depending only on the relative ratio $\bar{I}$ of the strong spin repulsion $I$ among fermions of opposite spins, to the characteristic energy of the particles in absence of interaction $E_{F}$; parameter $\bar{I}$ may be extracted from experiments, through the measurement of the paramagnetic susceptibility $\chi(T)$, extrapolated at $T=0 \mathrm{~K}$, and whose ratio to the density of states at the Fermi level gives the Stoner enhancement $(1-\bar{I})^{-1}$. The paramagnon model allowed to calculate, in particular, the $T$ dependence ${ }^{4}$ of $\chi(T)$ and to find that it is strongly enhanced by the spin fluctuations (the "paramagnons"), while $\chi(T=0)$ was not, compared to the mean-field (Stoner) value.

This was confirmed later on from the renormalization-group point of view: Owing to quantum effects at $T=0$, the critical exponents at $T=0$, when $\bar{I}=1$, of the $3 \mathrm{D}(d=3)$ paramagnon problem, assume their mean-field value; thus $\chi(T=0)$ diverges like $(I-\bar{I})^{-\gamma}$ with $\gamma=1$ as in mean field. ${ }^{6}$

The question whether such results still hold for lower dimensionalities $(d<3)$, in particular for $d=2$, was also considered. ${ }^{7}$ It appeared that simple conclusions can be reached for $d \neq 2$ because the magnetic instability $1 / I=\chi_{0}(q, \omega=0)$ occurs when $I$ increases for a unique value of $q$, for which $\chi_{0}$, the noninteracting susceptibility, is maximum [ $q=0$ for $d>2$, ferromagnetic instability; $q=2 k_{F}$ (where $k_{F}$ is the Fermi vector) for $d<2$, antiferromagnetic type of instability]. Instead, for $d=2$, the instability occurs for a continuum of $q$ values, $0 \leq q \leq 2 k_{F}$ due to the particular shape ${ }^{8}$ of the Lindhard function $\chi_{0}(q, \omega=0)$ at $d=2$, which is maximum and remains constant from $q=0$ to $q=2 k_{F}$. Therefore, compared to $d \neq 2$, the $d=2$ paramagnon problem appeared pathological. As was already determined in Ref. $7(\mathrm{~b})$, it may turn out to be a serious mistake, for $2 \mathrm{D}$ itinerant fermions at $T=0$, to approximate by constants the interaction coefficients in the Ginzburg-Landau-Wilson Lagrangian describing interacting paramagnons as is usually done in critical phenomena ${ }^{9}$ for localized spins at the transition temperature when vanishing momenta play the leading role. The reason for not doing so is that in paramagnon problems these coefficients correspond to interaction vertices given by multitail diagrams: closed fermion loops with an arbitrary number $n$ (even in absence of anisotropy) of paramagnons tails attached to them, and with $n$ increasing with the order of the corresponding term in the Wilson-type series expansion. The multitail diagrams were first studied by Brovman and Kagan ${ }^{10}$ for the $d=3$ electron-phonon problem, with the phonons as the tails. These authors showed that at $d=3$, such diagrams with static external fields are singular for some linear combination 


$$
\sum_{i} \bar{\alpha}_{i} q_{i}^{2}=4 k_{F}^{2}
$$

(the $q_{i}$ 's being the momenta of the external tails, and the $\bar{\alpha}_{i}$ 's being numerical coefficients between 0 and 1); they also showed that the degree of singularity increases with the number of tails. An analogous analysis for the $d=2$ paramagnon problem was sketched in Refs. 7(b) and 11; Ref. 11 pointed out more precisely that the singularities in $d=2$ are even stronger than in $d=3$. For $d=3$ paramagnon problems, these singularities are irrelevant as far as the magnetic instability is concerned, for which the relevant $q_{i}$ values are $q_{i} \sim 0$; then as in usual critical phenomena recalled above, it is reasonable to approximate by constants the various coefficients in the Wilson Lagrangian. Instead, for $d=2$, where all $q_{i}$ values from 0 to $2 k_{F}$ are equally relevant for the instability, the above singularities do play a key role and render the Wilson-type expansion questionable.

The purpose of the present paper is to analyze these singularities in detail and to examine the consequences for the critical behavior, as well as the $T$ dependence of the physical properties of 2D itinerant, nearly magnetic fermions. We first show in Sec. II that a calculation of $\chi(T)$ from first principles, taking into account paramagnon effects as was done successfully in $3 \mathrm{D}$ in Ref. 4, would be inadequate in $2 \mathrm{D}$ as it exhibits strong singularities. We then show in Sec. III why that is so, by analyzing the multitail diagrams which enter as basic ingredients in the calculation of $\chi(T)$; we show that these Brovman-Kagan diagrams are indeed highly singular. In Sec. IV we conclude that formal perturbation methods break down in the paramagnon problem in two dimensions. The question whether $2 \mathrm{D}$ itinerant fermion systems are close to a ferromagnetic instability or to an antiferromagnetic one thus appears to remain open. Furthermore, blind uses of the Landau theory which implicitly emphasize the role of small $q$ [through $\quad \epsilon(\overrightarrow{\mathrm{k}}+\overrightarrow{\mathrm{q}})-\epsilon(\overrightarrow{\mathrm{k}})=\overrightarrow{\mathrm{k}} \cdot \overrightarrow{\mathrm{q}}+q^{2} / 2$ $\left.\simeq k_{F} q \cos \theta\right]$ appears premature at this stage.

\section{FAILURE OF THE THERMODYNAMIC DERIVATION FOR $\chi(T)$ IN $d=2$}

In order to directly compare the $d=2$ case to the $d=3$, we first calculate $\chi(T)$ from first-principles thermo- dynamics as was done in Ref. 4. We follow the same procedure which proved to be successful in $d=3$. We write down the free energy with the same notations (we suppose the number of atoms per unit volume to be equal to 1 to simplify, and without any consequence for the main result, i.e., the existence of singular terms),

$$
F=G_{0}+\frac{I}{4}\left(1-\zeta^{2}\right)+\Delta F-\zeta B
$$

$\zeta$ is the difference of spins up and down, $B$ is a field "dressed" by the interactions,

$$
\begin{aligned}
& G_{0}=-\frac{1}{2} \chi_{\text {Pauli }} B^{2}, \\
& \chi_{\text {Pauli }}=2 \chi_{0}(q=0, \omega=0)=2 N\left(E_{F}\right),
\end{aligned}
$$

$N\left(E_{F}\right)$ is the density of states per spin direction at the Fermi level, $\chi_{0}(q, \omega)$ is the dynamic spin correlation function in absence of interaction, and $\Delta F$ is the contribution of the fluctuations. As in Ref. $4, B$ is determined by $(\partial F / \partial B)_{T, \zeta}=0$. This gives $-2 \chi_{0} B+\partial \Delta F / \partial B-\zeta=0$. On the other hand, $\partial B / \partial \xi$ is derived from $(\partial / \partial \zeta)(\partial F / \partial B)=0$, i.e.,

$$
\frac{\partial B}{\partial \zeta}\left[-2 \chi_{0}+\frac{\partial^{2} \Delta F}{\partial B^{2}}\right)=1
$$

Moreover, the static susceptibility in zero field is defined as

$$
\chi(H=0)=\mu_{m}^{2}\left(\frac{\partial^{2} F}{\partial \zeta^{2}}\right)^{-1},
$$

where $\mu_{m}$ is the magnetic moment and

$$
\frac{\partial^{2} F}{\partial \xi^{2}}=-\frac{I}{2}-\frac{\partial B}{\partial \xi} \text {. }
$$

With all these ingredients put together one gets

$$
\chi(H=0)=\frac{2 \chi_{0}-\partial^{2} \Delta F / \partial B^{2}}{1-\bar{I}+\frac{1}{2} I \partial^{2} \Delta F / \partial B^{2}}
$$

When the fluctuation contribution proves at low $T$ to be a perturbation, as is the case in $3 \mathrm{D}$ but not in $2 \mathrm{D}$, one is able to expand further in the limit of strong Stoner enhancement, $(1-\bar{I})^{-1} \gg 1$, to get

$$
\chi(T, H=0) \simeq \frac{2 \chi_{0}}{1-\bar{I}}\left[1-\frac{1}{2 \chi_{0}} \frac{1}{1-\bar{I}}\left(\frac{\partial^{2} \Delta F}{\partial B^{2}}\right]_{B=0}\right]=\chi(T=0, H=0)\left[1-\frac{2}{3} T_{F} \frac{1}{1-\bar{I}}\left[\frac{\partial^{2} \Delta F}{\partial B^{2}}\right]_{B=0}\right]
$$

This last form was given as such in Ref. 4 for $d=3$. In order to calculate $\partial^{2} \Delta F / \partial B^{2}$ we use the same closed diagrams as in Ref. 4 to write

$$
\Delta F=\frac{T}{2} \sum_{\overrightarrow{\mathrm{q}}, \omega}\left[\ln \left(1-I^{2} \chi_{0}^{++} \chi_{0}^{--}\right)+I^{2} \chi_{0}^{++} \chi_{0}^{--}+\ln \left(1-I \chi_{0}^{+-}\right)+I \chi_{0}^{+-}+\ln \left(1-I \chi_{0}^{-+}\right)+I \chi_{0}^{-+}\right]
$$

We are confined, as in Ref. 4 , to the most divergent terms in $1 /(1-\bar{I})$ since we are interested in the direct neighborhood of the instability $\bar{I} \rightarrow 1$; we also are confined to the lowest-order $T$ dependence; we then only need 


$$
\left(\frac{\partial^{2} \Delta F}{\partial B^{2}}\right]_{B=0}=T \sum_{\overrightarrow{\mathrm{q}}, \omega}\left\{-\left[\frac{\partial^{2} \operatorname{Re} \chi_{0}}{\partial \mu^{2}}-\left(\frac{\partial \operatorname{Re} \chi_{0}}{\partial \mu}\right)^{2}\right] \frac{I^{2}}{1-I^{2} \chi_{0}^{2}}-\left[\frac{\partial^{2} \operatorname{Re} \chi_{0}^{+-}}{\partial B^{2}}\right]_{B=0} \frac{I}{1-I \chi_{0}}-\left(\frac{\partial \operatorname{Re} \chi_{0}^{+-}}{\partial B}\right)_{B=0}^{2} \frac{I^{2}}{\left(1-I \chi_{0}\right)^{2}}\right\},
$$

where $\mu=k_{F}^{2} / 2$ (in a.u.), the chemical potential at $T=0$; its $T$ dependence will not appear in the calculation to the lowest order in $T$, as proven in Ref. 4. $\operatorname{Re} \chi_{0}^{\alpha \beta}$ is the real part of $\chi_{0}^{\alpha \beta}$.

We recall also from Ref. 4 that

$$
\chi_{0}^{\alpha \beta}=-\sum_{\overrightarrow{\mathrm{k}}} \frac{f_{\overrightarrow{\mathrm{k}}}^{\alpha}-f_{\overrightarrow{\mathrm{k}}+\overrightarrow{\mathrm{q}}}^{\beta}}{\omega+\xi_{\overrightarrow{\mathrm{k}}}^{\alpha}-\xi_{\overrightarrow{\mathrm{k}}+\overrightarrow{\mathrm{q}}}^{\beta}+i \eta \operatorname{sgn}\left(\xi_{\overrightarrow{\mathrm{k}}+\overrightarrow{\mathrm{q}}}^{\beta}-\xi_{\overrightarrow{\mathrm{k}}}^{\alpha}\right)},
$$

with $\xi_{\overrightarrow{\mathrm{k}}}^{\alpha \beta}=\xi_{\overrightarrow{\mathrm{k}}}+(\alpha, \beta) B, \alpha, \beta= \pm 1, \xi_{\overrightarrow{\mathrm{k}}}=k^{2} / 2-\mu$, and $\chi_{0}=\left(\chi_{0}^{\alpha \beta}\right)_{B=0}$.

So far, everything is unchanged compared to Ref. 4. We now use the fact that $d=2$. The calculation of the $\chi_{0}^{\alpha \beta}$ can be done rigorously and gives with a bit of tedious but straightforward algebra,

$$
\begin{aligned}
\operatorname{Re} \chi_{0}^{\alpha \beta}(q, \omega, B)=\frac{N\left(E_{F}\right)}{q^{2}}[ & \Theta\left(\left(\Omega-\epsilon_{q}\right)^{2}-q^{2} k_{F}^{2 \alpha}\right) \operatorname{sgn}\left(\Omega-\epsilon_{q}\right)\left\{\left[\left(\Omega-\epsilon_{q}\right)^{2}-q^{2} k_{F}^{2 \alpha}\right]^{1 / 2}-\left[\left(\Omega-\epsilon_{q}\right)^{2}\right]^{1 / 2}\right\} \\
& -\Theta\left(q^{2} k_{F}^{2 \alpha}-\left(\Omega-\epsilon_{q}\right)^{2}\right) \operatorname{sgn}\left(\Omega-\epsilon_{q}\right)\left[\left(\Omega-\epsilon_{q}\right)^{2}\right]^{1 / 2} \\
& -\Theta\left(\left(\Omega+\epsilon_{q}\right)^{2}-q^{2} k_{F}^{2 \beta}\right) \operatorname{sgn}\left(\Omega+\epsilon_{q}\right)\left\{\left[\left(\Omega+\epsilon_{q}\right)^{2}-q^{2} k_{F}^{2 \beta}\right]^{1 / 2}-\left[\left(\Omega+\epsilon_{q}\right)^{2}\right]^{1 / 2}\right\} \\
& \left.+\Theta\left(q^{2} k_{F}^{2 \beta}-\left(\Omega+\epsilon_{q}\right)^{2}\right) \operatorname{sgn}\left(\Omega+\epsilon_{q}\right)\left[\left(\Omega+\epsilon_{q}\right)^{2}\right]^{1 / 2}\right],
\end{aligned}
$$

$\operatorname{Im} \chi_{0}^{\alpha \beta}(q, \omega, B)=\frac{N\left(E_{F}\right)}{q^{2}}\left\{\left[q^{2} k_{F}^{2 \alpha}-\left(\Omega-\epsilon_{q}\right)^{2}\right]^{1 / 2} \Theta\left(q^{2} k_{F}^{2 \alpha}-\left(\Omega-\epsilon_{q}\right)^{2}\right)-\left[q^{2} k_{F}^{2 \beta}-\left(\Omega+\epsilon_{q}\right)^{2}\right]^{1 / 2} \Theta\left(q^{2} k_{F}^{2 \beta}-\left(\Omega+\epsilon_{q}\right)^{2}\right)\right\}$,

where $N\left(E_{F}\right)=(2 \pi)^{-1}$ for $d=2, \epsilon_{q}=q^{2} / 2, \Omega=\omega+(\alpha-\beta) B, k_{F}^{2}(\alpha, \beta)=k_{F}^{2}-2(\alpha, \beta) B, \Theta(x)=1$ for $x>0$ and $\Theta(x)=0$ for $x<0$, and $\operatorname{sgn} x=+1$ for $x>0$ and $\operatorname{sgn} x=-1$ for $x<0$. In further calculations we use

$$
\begin{aligned}
& {\left[\frac{\partial \operatorname{Re} \chi_{0}^{++}}{\partial B}+\frac{\partial \operatorname{Re} \chi_{0}^{--}}{\partial B}\right]_{B=0}=0,} \\
& {\left[\frac{\partial^{2} \operatorname{Re} \chi_{0}^{++}}{\partial B^{2}}+\frac{\partial^{2} \operatorname{Re} \chi_{0}^{--}}{\partial B^{2}}\right]_{B=0}=2 \frac{\partial^{2} \operatorname{Re} \chi_{0}}{\partial \mu^{2}},} \\
& \operatorname{Re} \chi_{0}^{-+}(q, \omega, B)=\operatorname{Re} \chi_{0}^{+-}(q, \omega,-B) .
\end{aligned}
$$

We also use

$$
\left(\frac{1}{4} \frac{\partial^{2} \operatorname{Re} \chi_{0}}{\partial \mu^{2}}\right)_{\omega=0}=4 N\left(E_{F}\right) \frac{\Theta\left(q^{2}-4 k_{F}^{2}\right)}{q^{4}\left(1-4 k_{F}^{2} / q^{2}\right)^{3 / 2}},
$$

with $\delta(x)=\partial \Theta(x) / \partial x$.

Then putting (9) back into (5) shows clearly that strong singularities will arise for $q=2 k_{F}$ and survive even after integrations. A few comments take place here.

(1) As already announced, the fluctuation contribution to the calculation of the susceptibility cannot be considered as a perturbation as in the 3D case of Ref. 4.

(2) Aside from the expected divergences in $1 /(1-\bar{I}$ (when $\bar{I} \rightarrow 1$ ) common to the $3 \mathrm{D}$ and $2 \mathrm{D}$ cases and characteristic of strong spin fluctuations, extra divergences arise in $2 \mathrm{D}$; the strongest originates in $\partial^{2} \chi_{0} / \partial \mu^{2}$ as shown above, and is linked to the very structure of the four-tail diagram. Indeed one of the lowest paramagnon corrections to $\chi_{0}\left(\chi_{0}\right.$ itself being a two-tail diagram, Fig. 1), involves one paramagnon insertion (Fig. 2) which brings in two extra vertices in that first-order correction shown on Fig. 3; therefore, such first-order correction to $\chi_{0}$ involves a four-tail diagram (Fig. 4) with two of the tails attached to one another to form the inserted paramagnon (Fig. 5). On the other hand, as will be 


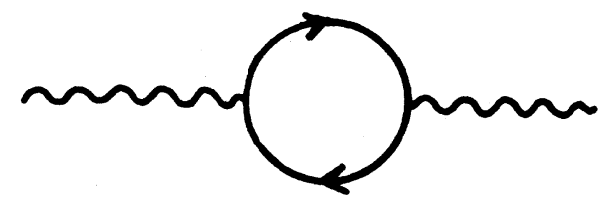

FIG. 1. Bare-bubble diagram for the noninteracting susceptibility $\chi_{0}$.

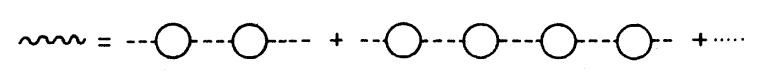

FIG. 2. One of the possible paramagnon propagators: the geometric series of even numbers of bare bubbles linked by interactions (dotted lines), entering as a vertex correction in Figs. 3 and 5.

shown later, the $n$th derivative of the two-tail diagram $\left(n=2\right.$ in $\left.\partial^{2} \chi_{0} / \partial \mu^{2}\right)$ has the same singularities that the $(n+2)$-tail diagram, i.e., the four-tail one here.

More generally the singularities characteristic of 2D have their sources in the structure of the $n$ th-tail diagram formed by a closed fermion loop with $n$ vertices where the $n$ external tails are attached,

$$
\begin{aligned}
J^{(n)}\left(\overrightarrow{\mathrm{q}}_{1}, \ldots, \overrightarrow{\mathrm{q}}_{n} ; \omega_{1}, \ldots, \omega_{n}\right)=-T \sum_{\overrightarrow{\mathrm{p}}, \epsilon}\left[G_{0}(\overrightarrow{\mathrm{p}}, \epsilon) G_{0}\left(\overrightarrow{\mathrm{p}}+\overrightarrow{\mathrm{q}}_{1}, \epsilon+\omega_{1}\right) \cdots G_{0}\left(\overrightarrow{\mathrm{p}}+\overrightarrow{\mathrm{q}}_{1}+\cdots+\overrightarrow{\mathrm{q}}_{n-1}, \epsilon+\omega_{1}+\cdots+\omega_{n-1}\right)\right. \\
\left.\times \delta\left(\sum_{i=1}^{n} \overrightarrow{\mathrm{q}}_{i}\right) \delta\left(\sum_{i=1}^{n} \omega_{i}\right)\right]
\end{aligned}
$$

where $G_{0}(\overrightarrow{\mathrm{p}}, \epsilon)$ is the fermion Green's function. Whatever the tails are made of (paramagnons, phonons, extra fields, etc.) the above closed loop with $n$ vertices, which itself may be regarded as one interaction vertex among the $n$ tails, is the crucial singular quantity in 2D. In the next section we analyze in detail the four-tail ring diagram.

\section{SINGULARITIES OF THE MULTITAIL RING DIAGRAM}

In this section we examine the singular behavior of multitail ring diagrams by generalizing the method of Brovman and Kagan ${ }^{10}$ to $d$ dimensions and to dynamic legs in the four-tail diagram. Although the calculation here is performed at $T=0 \mathrm{~K}$, the results of Sec. II show that the singularities persist at finite $T$. Because of momentum and energy conservation the $m$-tail ring diagram involves $m-1$ external momenta $\overrightarrow{\mathrm{q}}_{1}, \overrightarrow{\mathrm{q}}_{2}, \ldots, \overrightarrow{\mathrm{q}}_{m-1}$ and the corresponding energies $\omega_{1}, \omega_{2}, \ldots, \omega_{m-1}$.

The method of Ref. 10 allows us to tell if a singularity may occur for some values of the external momenta and energies, and to evaluate the asymptotic behavior close to the singular point. In Sec. III A we examine the four-tail diagram with dynamic external tails of Fig. 4, for arbitrary dimensionality $d$. In Sec. III B we look for the static $2 n$-tail diagram with arbitrary momenta which is the analogous in 2D to what Brovman and Kagan studied in 3D.

\section{A. Four-tail diagram with finite $q_{i}$ 's and two dynamic legs}

Our purpose here is to analyze more precisely the behavior of one of the lowest-order diagrams entering in the calculation of the susceptibility, i.e., the diagram displayed in Fig. 3 and related to the four-tail diagram as indicated in Fig. 5. We first present a general calculation for arbitrary dimensionality $d$ then we will apply it for $d=2$.

\section{Calculation for arbitrary dimensionality $d$}

We recall that the bare fermion propagator is, at $T=0 \mathrm{~K}$ and for real continuous energies,

$$
G_{0}(\overrightarrow{\mathrm{p}}, \epsilon)=\frac{1}{\epsilon-\xi_{\overrightarrow{\mathrm{p}}}+i \eta \operatorname{sgn}\left(|\overrightarrow{\mathrm{p}}|-p_{F}\right)}=\frac{1}{\epsilon-\xi_{\overrightarrow{\mathrm{p}}}+i \eta \operatorname{sgn}(\epsilon)},
$$

with the notation of Sec. II. The diagram of Fig. 3 is then expressed in $d$ dimensions as

$$
\chi_{1}\left(\overrightarrow{\mathrm{q}}_{1}, \omega_{1}\right)=\iint \frac{d \overrightarrow{\mathrm{q}}_{2} d \overrightarrow{\mathrm{q}}_{3} d \omega_{2} d \omega_{3}}{(2 \pi)^{2 d+2}} \Pi\left(\overrightarrow{\mathrm{q}}_{2}, \omega_{2}\right) J_{4}\left(q_{1}, q_{2}, q_{3} ; \omega_{1}, \omega_{2}, \omega_{3}\right) \delta\left(\overrightarrow{\mathrm{q}}_{3}-\overrightarrow{\mathrm{q}}_{1}-\overrightarrow{\mathrm{q}}_{2}\right) \delta\left(\omega_{3}-\omega_{1}-\omega_{2}\right),
$$

where $J_{4}$ is the dynamic four-tail ring diagram of Fig. 4 ,

$$
J_{4}\left(\overrightarrow{\mathrm{q}}_{1}, \overrightarrow{\mathrm{q}}_{2}, \overrightarrow{\mathrm{q}}_{3} ; \omega_{1}, \omega_{2}, \omega_{3}\right)=-i \int \frac{d \overrightarrow{\mathrm{p}} d \epsilon}{(2 \pi)^{d+1}} G_{0}(\overrightarrow{\mathrm{p}}, \epsilon) G_{0}\left(\overrightarrow{\mathrm{p}}+\overrightarrow{\mathrm{q}}_{1}, \epsilon+\omega_{1}\right) G_{0}\left(\overrightarrow{\mathrm{p}}+\overrightarrow{\mathrm{q}}_{3}, \epsilon+\omega_{3}\right) G_{0}\left(\overrightarrow{\mathrm{p}}+\overrightarrow{\mathrm{q}}_{2}, \epsilon+\omega_{2}\right)
$$

while $\Pi\left(\overrightarrow{\mathrm{q}}_{2}, \omega_{2}\right)$ is the appropriate fluctuation or paramagnon propagator, 


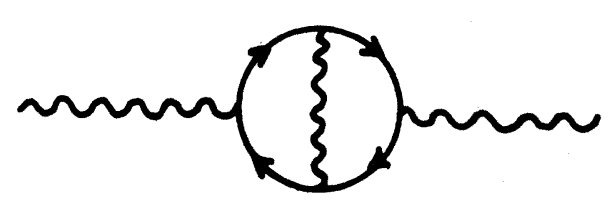

FIG. 3. One of the lowest-order correction to the bare bubble of Fig. 1, with the paramagnon insertion of Fig. 2 exchanged between the two bare fermion lines of the bare bubble.

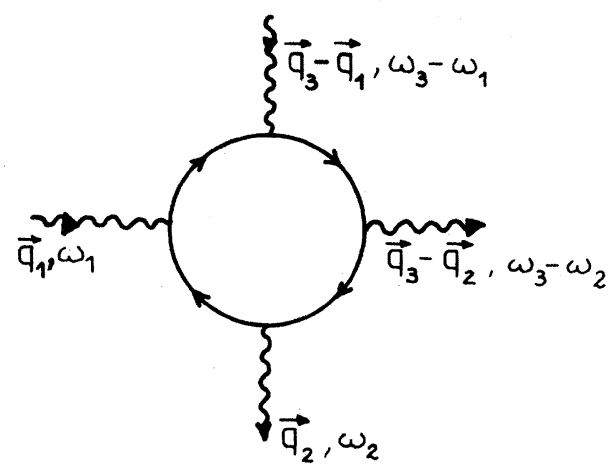

FIG. 4. Four-tail Brovman-Kagan-type diagram: one closed fermion loop with four fluctuations attached to it; such a diagram represents, as well, the lowest-order interaction potential (i.e., the closed loop) among two fluctuations.

$$
\Pi\left(\overrightarrow{\mathrm{q}}_{2}, \omega_{2}\right)=\frac{I^{3} \chi_{0}^{2}\left(\overrightarrow{\mathrm{q}}_{2}, \omega_{2}\right)}{I-I^{2} \chi_{0}^{2}\left(\overrightarrow{\mathrm{q}}_{2}, \omega_{2}\right)} .
$$

To analyze the singularities of $J_{4}$ in Eq. (13) we generalize the method of Brovman and $\mathrm{Kagan}^{10}$ to arbitrary dimensionality $d$ and to dynamic tails. We are restricted, however, for simplicity, to the static case for $\chi_{1}$,

$$
\omega_{1}=0 \text {, }
$$

and to

$$
\bar{J}_{4}\left(\overrightarrow{\mathrm{q}}_{1}, \overrightarrow{\mathrm{q}}_{2} ; \omega_{2}\right)=\int \frac{d \overrightarrow{\mathrm{q}}_{3} d \omega_{3}}{(2 \pi)^{d+1}} J_{4}\left(q_{1}, q_{2}, q_{3} ; 0, \omega_{2}, \omega_{3}\right) \delta\left(\overrightarrow{\mathrm{q}}_{3}-\overrightarrow{\mathrm{q}}_{1}-\overrightarrow{\mathrm{q}}_{2}\right) \delta\left(\omega_{3}-\omega_{2}\right),
$$

which will enter in

$$
\chi_{1}\left(\overrightarrow{\mathrm{q}}_{1}, 0\right)=\iint \frac{d \overrightarrow{\mathrm{q}}_{2} d \omega_{2}}{(2 \pi)^{d+1}} \Pi\left(\overrightarrow{\mathrm{q}}_{2}, \omega_{2}\right) \bar{J}_{4}\left(\overrightarrow{\mathrm{q}}_{1}, \overrightarrow{\mathrm{q}}_{2} ; \omega_{2}\right) .
$$

We use the Feynman parametrization,

$$
\frac{1}{a_{0} a_{1} a_{2} a_{3}}=3 ! \int_{0}^{1} \frac{d \alpha_{1} d \alpha_{2} d \alpha_{3} \Theta\left(1-\alpha_{1}-\alpha_{2}-\alpha_{3}\right)}{\left[\left\lfloor 1-\sum_{i=1}^{3} \alpha_{i}\right] a_{0}+\sum_{i=1}^{3} \alpha_{i} a_{i}\right]^{4}},
$$

in order to write $\bar{J}_{4}\left(\overrightarrow{\mathrm{q}}_{1}, \overrightarrow{\mathrm{q}}_{2} ; \omega_{2}\right)$ with the change of variable,

$$
\begin{aligned}
& \overrightarrow{\mathrm{p}} \rightarrow \overrightarrow{\mathrm{p}}-\sum_{i=1}^{3} \alpha_{i} \overrightarrow{\mathrm{q}}_{i}, \\
& \bar{J}_{4}\left(\overrightarrow{\mathrm{q}}_{1}, \overrightarrow{\mathrm{q}}_{2} ; \omega_{2}\right)=\frac{K_{d}}{2(2 \pi)^{d}} \int_{0}^{1} d \alpha_{1} d \alpha_{2} d \alpha_{3} \Theta\left[1-\sum_{i=1}^{3} \alpha_{i}\right] L\left(\alpha_{i}, \overrightarrow{\mathrm{q}}_{i}, \omega_{2}\right),
\end{aligned}
$$

where $K_{d}=2 \pi^{d / 2}[\Gamma(d / 2)]^{-1}$ is the surface of the unit sphere and

$$
L\left(\alpha_{i}, \overrightarrow{\mathrm{q}}_{i} ; \omega_{2}\right)=3 ! \int_{0}^{\infty} p^{d-2} d\left(p^{2}\right) \int_{-\infty}^{+\infty} \frac{d \epsilon}{2 i \pi} \frac{1}{\left\{G_{0}^{-1}(\overrightarrow{\mathrm{p}}, \epsilon)+f / 2+i \eta\left(\alpha_{2}+\alpha_{3}\right)\left[\operatorname{sgn}\left(\epsilon+\omega_{2}\right)-\operatorname{sgn}(\epsilon)\right]\right\}^{4}},
$$

with

$$
f=f\left(\alpha_{i}, \overrightarrow{\mathrm{q}}_{i} ; \omega_{2}\right)=\left(\sum_{i} \alpha_{i} \overrightarrow{\mathrm{q}}_{i}\right)^{2}-\sum_{i} \alpha_{i} q_{i}^{2}+2\left(\alpha_{2}+\alpha_{3}\right) \omega_{2} .
$$

Taking into account that for $\omega_{2}>0$,

$$
\operatorname{sgn}\left(\epsilon+\omega_{2}\right)-\operatorname{sgn}(\epsilon)= \begin{cases}2 & \text { if }-\omega_{2}<\epsilon<0 \\ 0 & \text { otherwise }\end{cases}
$$


the frequency integral in Eq. (21) is performed by splitting the region of integration in $-\infty<\epsilon<-\omega_{2},-\omega_{2}<\epsilon<0$, and $0<\epsilon<\infty$ with the result,

$$
\begin{aligned}
L\left(\alpha_{i}, \overrightarrow{\mathrm{q}}_{i} ; \omega_{2}\right)=\frac{2}{\pi} \int_{0}^{\infty} p^{d-2} d\left(p^{2}\right)[ & \Theta\left(\frac{1}{2}-\alpha_{2}-\alpha_{3}\right) \operatorname{Im} \frac{1}{\left(\mu-p^{2} / 2+f / 2+i \eta\right)^{3}} \\
& \left.+\Theta\left(\alpha_{2}+\alpha_{3}-\frac{1}{2}\right) \operatorname{Im} \frac{1}{\left(\mu-p^{2} / 2+f / 2-\omega_{2}+i \eta\right)^{3}}\right],
\end{aligned}
$$

where for $\eta$ infinitesimal we replaced $\left(2 \alpha_{2}+2 \alpha_{3}-1\right) \eta$ by $\eta \operatorname{sgn}\left(\alpha_{2}+\alpha_{3}-\frac{1}{2}\right)$. We use the formal identity,

$$
\operatorname{Im} \frac{1}{(\mu+x+i \eta)^{3}}=\frac{1}{2} \frac{\partial^{2}}{\partial \mu^{2}} \operatorname{Im} \frac{1}{(\mu+x+i \eta)}=-\frac{\pi}{2} \frac{\partial^{2}}{\partial \mu^{2}} \delta(\mu+x),
$$

to perform the integral in Eq. (24). It is clear that what we obtain by this method is only $\operatorname{Re} \bar{J}_{4}\left(\vec{q}_{1}, \overrightarrow{\mathrm{q}}_{2} ; \omega_{2}\right)$ that reads, from Eq. (20),

$$
\operatorname{Re} \bar{J}_{4}\left(\overrightarrow{\mathrm{q}}_{1}, \overrightarrow{\mathrm{q}}_{2} ; \omega_{2}\right)=-\frac{K_{d}}{(2 \pi)^{d}} \frac{\partial^{2}}{\partial \mu^{2}}\left[\psi_{a}\left(\overrightarrow{\mathrm{q}}_{1}, \overrightarrow{\mathrm{q}}_{2} ; \omega_{2}\right)+\psi_{b}\left(\overrightarrow{\mathrm{q}}_{1}, \overrightarrow{\mathrm{q}}_{2} ; \omega_{2}\right)\right],
$$

with

$$
\psi_{a, b}\left(\overrightarrow{\mathrm{q}}_{1}, \overrightarrow{\mathrm{q}}_{2} ; \omega_{2}\right)=\left[\psi_{a, b}\left(\overrightarrow{\mathrm{q}}_{i}, \omega_{2}\right)\right]_{\overrightarrow{\mathrm{q}}_{3}=\overrightarrow{\mathrm{q}}_{1}+\overrightarrow{\mathrm{q}}_{2}},
$$

and

$$
\begin{aligned}
& \psi_{a}\left(\overrightarrow{\mathrm{q}}_{i}, \omega_{2}\right)=\int_{0}^{1}\left[\prod_{i} d \alpha_{i}\right]^{\Theta}\left[1-\sum_{i} \alpha_{i}\right]^{\Theta}\left(\frac{1}{2}-\alpha_{2}-\alpha_{3}\right) \Theta(\mu+f / 2)(\mu+f / 2)^{(d-2) / 2}, \\
& \psi_{b}\left(\overrightarrow{\mathrm{q}}_{i}, \omega_{2}\right)=\int_{o}^{1}\left[\prod_{i} d \alpha_{i}\right]^{\Theta}\left[1-\sum_{i} \alpha_{i}\right]^{\Theta}\left(-\frac{1}{2}+\alpha_{2}+\alpha_{3}\right) \Theta\left(\mu+f / 2-\omega_{2}\right)\left(\mu+f / 2-\omega_{2}\right)^{(d-2) / 2} .
\end{aligned}
$$

To analyze the asymptotic singular behavior of $\psi_{a, b}\left(\vec{q}_{1}, \vec{q}_{2} ; \omega_{2}\right)$ in Eq. (27) we first change variables

$$
\alpha_{i}=\bar{\alpha}_{i}+\beta_{i} \text { with } i=1,2,3
$$

in Eqs. (28) in order to eliminate the linear terms ${ }^{10}$ in $f\left(\beta_{i}, \vec{q}_{i} ; \omega_{2}\right)$ of Eq. (22). It turns out that the $\bar{\alpha}_{i}$ should satisfy the equations

$$
\begin{aligned}
& \overrightarrow{\mathrm{q}}_{1} \cdot \sum_{j=1}^{3} \bar{\alpha}_{j} \overrightarrow{\mathrm{q}}_{j}=\frac{1}{2} q_{1}^{2}, \\
& \overrightarrow{\mathrm{q}}_{i} \cdot \sum_{j=1}^{3} \bar{\alpha}_{j} \overrightarrow{\mathrm{q}}_{j}=\frac{1}{2} q_{i}^{2}-\omega_{2} \text { for } i=2,3,
\end{aligned}
$$

and one obtains by introducing Eqs. (29) and (30) into Eq. (22),

$$
\begin{aligned}
& f=\sum_{i, j} \beta_{i} \beta_{j} \overrightarrow{\mathrm{q}}_{i} \cdot \overrightarrow{\mathrm{q}}_{j}-\Delta\left(\omega_{2}\right)-2 \mu \\
& \Delta\left(\omega_{2}\right)=\frac{1}{2} \sum_{i} \bar{\alpha}_{i} q_{i}^{2}-\left(\bar{\alpha}_{2}+\bar{\alpha}_{3}\right) \omega_{2}-k_{F}^{2} .
\end{aligned}
$$

Satisfying the system of Eqs. (30) is sufficient for the existence of a singularity, as was shown in Ref. 10 . Moreover, the external momenta $\overrightarrow{\mathrm{q}}_{i}$ and the frequency $\omega_{2}$ must be such that the solutions $\bar{\alpha}_{i}$ fall within the range of integration: $\bar{\alpha}_{i}>0$, $1-\sum_{i} \bar{\alpha}_{i}>0$. When the external momenta are not independent, the Gram determinant $\left|\overrightarrow{\mathrm{q}}_{i} \cdot \overrightarrow{\mathrm{q}}_{j}\right|=0$, and Eqs. (30) have a solution only when some restrictive condition among the $\vec{q}_{i}$ is satisfied. In particular, we find for $\vec{q}_{3}=\vec{q}_{1}+\vec{q}_{2}$ that Eqs. (30) are consistent only if $\vec{q}_{1} \cdot \overrightarrow{\mathrm{q}}_{2}=0$. In this case they reduce to two equations for $\bar{\alpha}_{1}+\bar{\alpha}_{3}, \bar{\alpha}_{2}+\bar{\alpha}_{3}$, with solutions

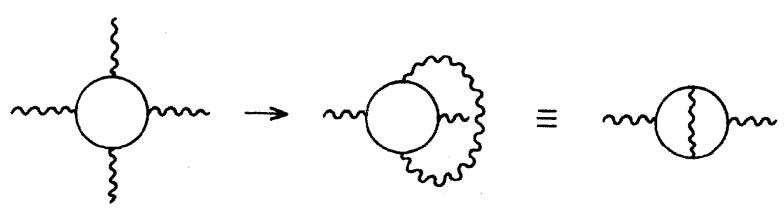

FIG. 5. How the four-tail diagram generates one of the lowest-order vertex corrections to $\chi_{0}$ displayed in Fig. 3 .

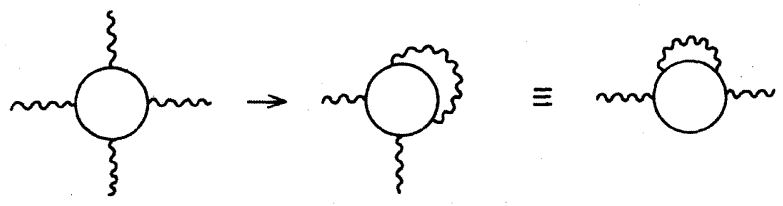

FIG. 6. How the four-tail diagram generates another lowestorder correction (self-energy correction) to $\chi_{0}$. 


$$
\begin{aligned}
& \bar{\alpha}_{1}\left(\omega_{2}\right)+\bar{\alpha}_{3}\left(\omega_{2}\right)=\frac{1}{2}, \\
& \bar{\alpha}_{2}\left(\omega_{2}\right)+\bar{\alpha}_{3}\left(\omega_{2}\right)=\frac{1}{2}-\frac{\omega_{2}}{q_{2}^{2}}, \\
& \overrightarrow{\mathrm{q}}_{1} \cdot \overrightarrow{\mathrm{q}}_{2}=0,
\end{aligned}
$$

where we made explicit the dependence of the solutions on $\omega_{2}$. It follows from Eqs. (27), (28), (31), and (33) that

$$
\begin{aligned}
\psi_{a}\left(\overrightarrow{\mathrm{q}}_{1}, \overrightarrow{\mathrm{q}}_{2}, \omega_{2}\right)=2^{1-d / 2} \int & {\left[\prod_{i} d \beta_{i} \Theta\left(\bar{\alpha}_{i}\left(\omega_{2}\right)+\beta_{i}\right)\right] \Theta\left[1-\sum_{i} \bar{\alpha}_{i}\left(\omega_{2}\right)-\sum_{i} \beta_{i}\right] } \\
\times & \times \Theta\left[\frac{\omega_{2}}{q_{2}^{2}}-\beta_{2}-\beta_{3}\right] \Theta\left(x_{1}^{2}+x_{2}^{2}-\Delta\left(\omega_{2}\right)\right)\left[x_{1}^{2}+x_{2}^{2}-\Delta\left(\omega_{2}\right)\right]^{(d-2) / 2},
\end{aligned}
$$

where

$$
\begin{aligned}
& x_{1}=\left(\beta_{1}+\beta_{3}\right) q_{1}, \\
& x_{2}=\left(\beta_{2}+\beta_{3}\right) q_{2}, \\
& \Delta\left(\omega_{2}\right)=\frac{1}{4}\left[q_{1}^{2}+q_{2}^{2}\left(1-2 \omega_{2} / q_{2}^{2}\right)^{2}-4 k_{F}^{2}\right]=\Delta\left(-\omega_{2}\right)-2 \omega_{2} .
\end{aligned}
$$

In order to evaluate $\psi_{b}\left(\overrightarrow{\mathrm{q}}_{1}, \overrightarrow{\mathrm{q}}_{2}, \omega_{2}\right)$ in Eq. (28b) we first change to integration variables $\gamma_{i}$ defined by

$$
\begin{aligned}
& \bar{\alpha}_{1}\left(\omega_{2}\right)+\beta_{1}=\bar{\alpha}_{3}\left(-\omega_{2}\right)+\gamma_{3}, \\
& \bar{\alpha}_{3}\left(\omega_{2}\right)+\beta_{3}=\bar{\alpha}_{1}\left(-\omega_{2}\right)+\gamma_{1}, \\
& \beta_{2}+\beta_{3}=-\left(\gamma_{2}+\gamma_{3}\right),
\end{aligned}
$$

from where it follows, using Eqs. (33),

$$
\begin{aligned}
& \bar{\alpha}_{2}\left(\omega_{2}\right)+\beta_{2}=1-\sum_{i=1}^{3} \bar{\alpha}_{i}\left(-\omega_{2}\right)-\sum_{i=1}^{3} \gamma_{i}, \\
& \bar{\alpha}_{2}\left(-\omega_{2}\right)+\gamma_{2}=1-\sum_{i=1}^{3} \bar{\alpha}_{i}\left(\omega_{2}\right)-\sum_{i=1}^{3} \beta_{i}, \\
& \beta_{1}+\beta_{3}=\gamma_{1}+\gamma_{3} .
\end{aligned}
$$

By introducing Eqs. (37) and (38), together with the last equality in (36), into Eq. (28b), one obtains the result,

$$
\begin{aligned}
\psi_{b}\left(\overrightarrow{\mathrm{q}}_{1}, \overrightarrow{\mathrm{q}}_{2} ; \omega_{2}\right)=2^{1-d / 2} \int & {\left[\prod_{i} d \gamma_{i} \Theta\left(\bar{\alpha}_{i}\left(-\omega_{2}\right)+\gamma_{i}\right)\right] \Theta\left[1-\sum_{i} \bar{\alpha}_{i}\left(-\omega_{2}\right)-\sum_{i} \gamma_{i}\right] \Theta\left[-\frac{\omega_{2}}{q_{2}^{2}}-\gamma_{2}-\gamma_{3}\right] } \\
& \times \Theta\left(x_{1}^{2}+x_{2}^{2}-\Delta\left(-\omega_{2}\right)\right)\left[x_{1}^{2}+x_{2}^{2}-\Delta\left(-\omega_{2}\right)\right]^{(d-2) / 2},
\end{aligned}
$$

with $x_{1}, x_{2}$ as in Eq. (35) with $\gamma_{i}$ in place of $\beta_{i}$. By comparing Eq. (39) with Eq. (34) one obtains

$$
\psi_{b}\left(\overrightarrow{\mathrm{q}}_{1}, \overrightarrow{\mathrm{q}}_{2} ; \omega_{2}\right) \equiv \psi_{a}\left(\overrightarrow{\mathrm{q}}_{1}, \overrightarrow{\mathrm{q}}_{2} ;-\omega_{2}\right) \equiv \psi\left(\overrightarrow{\mathrm{q}}_{1}, \overrightarrow{\mathrm{q}}_{2} ;-\omega_{2}\right)
$$

and from Eq. (26),

$$
\operatorname{Re} \bar{J}_{4}\left(\overrightarrow{\mathrm{q}}_{1}, \overrightarrow{\mathrm{q}}_{2} ; \omega_{2}\right)=-\frac{K_{d}}{(2 \pi)^{d}} \frac{\partial^{2}}{\partial \mu^{2}}\left[\psi\left(\overrightarrow{\mathrm{q}}_{1}, \overrightarrow{\mathrm{q}}_{2} ; \omega_{2}\right)+\psi\left(\overrightarrow{\mathrm{q}}_{1}, \overrightarrow{\mathrm{q}}_{2} ;-\omega_{2}\right)\right],
$$

where $\psi \equiv \psi_{a}$ in Eq. (34).

While $\operatorname{Re} \bar{J}_{4}\left(\overrightarrow{\mathrm{q}}_{1}, \overrightarrow{\mathrm{q}}_{2} ; \omega_{2}\right)$ in Eq. (41) is an even function of the frequency, the imaginary part $\operatorname{Im} \bar{J}_{4}\left(\overrightarrow{\mathrm{q}}_{1}, \overrightarrow{\mathrm{q}}_{2} ; \omega_{2}\right)$ is an odd function which is obtained by analytic continuation into the complex $\omega$ plane.

If we define the complex function,

$$
\bar{\psi}\left(\overrightarrow{\mathrm{q}}_{1}, \overrightarrow{\mathrm{q}}_{2} ; \omega_{2}\right)=\frac{1}{i \pi} \int_{-\infty}^{+\infty} d \omega^{\prime} \frac{1}{\omega^{\prime}-\omega_{2}-i \eta}\left[\psi\left(\overrightarrow{\mathrm{q}}_{1}, \overrightarrow{\mathrm{q}}_{2} ; \omega^{\prime}\right)+\psi\left(\overrightarrow{\mathrm{q}}_{1}, \overrightarrow{\mathrm{q}}_{2} ;-\omega^{\prime}\right)\right],
$$


then

$$
\bar{J}_{4}\left(\overrightarrow{\mathrm{q}}_{1}, \overrightarrow{\mathrm{q}}_{2} ; \omega_{2}\right)=-\frac{K_{d}}{(2 \pi)^{d}} \frac{\partial^{2}}{\partial \mu^{2}} \bar{\psi}\left(\overrightarrow{\mathrm{q}}_{1}, \overrightarrow{\mathrm{q}}_{2} ; \omega_{2}\right),
$$

and the imaginary part is given by the Kramers-Kronig relation,

$$
\operatorname{Im} \bar{J}_{4}\left(\overrightarrow{\mathrm{q}}_{1}, \overrightarrow{\mathrm{q}}_{2} ; \omega_{2}\right)=-\frac{1}{\pi} \mathrm{P} \int_{-\infty}^{+\infty} \frac{1}{\omega^{\prime}-\omega_{2}} \operatorname{Re} \bar{J}_{4}\left(\overrightarrow{\mathrm{q}}_{1}, \overrightarrow{\mathrm{q}}_{2} ; \omega^{\prime}\right) d \omega^{\prime} .
$$

We discuss now the asymptotic behavior of $\psi\left(\overrightarrow{\mathrm{q}}_{1}, \overrightarrow{\mathrm{q}}_{2} ; \omega_{2}\right)$ in Eq. (34) close to its singular point $\Delta\left(\omega_{2}\right)=0$. It was pointed out in Ref. 10 that the singular contribution of this integral, if any, comes only from the lower integration limit and, in general, it would be of the type $\left|\Delta\left(\omega_{2}\right)\right|^{\alpha}$ or $\left|\Delta\left(\omega_{2}\right)\right|^{\beta} \ln \left|\Delta\left(\omega_{2}\right)\right|$. This may introduce divergences in $\bar{J}_{4}\left(\vec{q}_{1}, \vec{q}_{2} ; \omega_{2}\right)$ in Eq. (43) because $\partial / \partial \mu=-\partial / \partial \Delta$ from Eq. (36).

We call now $\psi_{\text {sing }}\left(\overrightarrow{\mathrm{q}}_{1}, \overrightarrow{\mathrm{q}}_{2} ; \omega_{2}\right)$ the singular part of the integral in Eq. (34) when the upper limit is replaced by an arbitrary cutoff $\Lambda$. We distinguish three cases.

(a) $q_{1}>0, q_{2}>0$, and $\overrightarrow{\mathrm{q}}_{1} \cdot \overrightarrow{\mathrm{q}}_{2}=0$. For $\left|\Delta\left(\omega_{2}\right)\right|<\omega_{2}^{2} / q_{2}^{2}$ we introduce polar coordinates with $\tau=\left(x_{1}^{2}+x_{2}^{2}\right)^{1 / 2}$ and $x_{1,2}$ as defined in Eq. (35) to obtain

$$
\psi_{\text {sing }}\left(\overrightarrow{\mathrm{q}}_{1}, \overrightarrow{\mathrm{q}}_{2} ; \omega_{2}\right) \approx \frac{1}{q_{1} q_{2}} \int_{0}^{\Lambda} d \tau \tau \Theta\left(\tau^{2}-\Delta\left(\omega_{2}\right)\right)\left[\tau^{2}-\Delta\left(\omega_{2}\right)\right]^{d / 2-1},
$$

with the result,

$$
\psi_{\text {sing }}\left(\overrightarrow{\mathrm{q}}_{1}, \overrightarrow{\mathrm{q}}_{2} ; \omega_{2}\right) \approx\left\{\begin{array}{l}
\left|\Delta\left(\omega_{2}\right)\right|^{d / 2} \text { if } \Delta\left(\omega_{2}\right)<0 \\
0 \text { if } \Delta\left(\omega_{2}\right)>0
\end{array}\right.
$$

In particular, for $d=2$ there is no singularity.

(b) $q_{1}=0, q_{2}>0$, and $\overrightarrow{\mathrm{q}}_{1} \cdot \overrightarrow{\mathrm{q}}_{2}=0$. This case corresponds to a contribution to the uniform static susceptibility. From Eqs. (34) and (35) we get

$$
\psi_{\text {sing }}\left(\overrightarrow{\mathrm{q}}_{1}, \overrightarrow{\mathrm{q}}_{2} ; \omega_{2}\right) \approx \frac{1}{q_{2}} \int_{-\bar{x}_{2}}^{\Lambda} d y \Theta\left(y^{2}-\Delta\right)\left(y^{2}-\Delta\right)^{d / 2-1},
$$

where $\bar{x}_{2}=q_{2} / 2\left[1-\left(2 \omega_{2} / q_{2}^{2}\right)\right]$ from Eq. (33).

Now we obtain for $d=2$,

$$
\psi_{\text {sing }}\left(\overrightarrow{\mathrm{q}}_{1}, \overrightarrow{\mathrm{q}}_{2} ; \omega_{2}\right) \approx\left\{\begin{array}{l}
{\left[\Delta\left(\omega_{2}\right)\right]^{1 / 2} \text { if } \Delta\left(\omega_{2}\right)>0} \\
0 \text { if } \Delta\left(\omega_{2}\right)<0 .
\end{array}\right.
$$

For $2<d<3$ we use the identity,

$$
(d-1) \int d y\left(y^{2}-\Delta\right)^{d / 2-1}=y\left(y^{2}-\Delta\right)^{(d / 2-1)}-(d-2) \Delta \int d y\left(y^{2}-\Delta\right)^{d / 2-2},
$$

in Eq. (47). The first term in the right-hand side of Eq. (49) is not singular, for either sign of $\Delta\left(\omega_{2}\right)$. In the second term one can let the upper and lower integration limits go to $\pm \infty$ because the integral is convergent with the result for $2<d<3$,

$$
\psi_{\text {sing }}\left(\overrightarrow{\mathrm{q}}_{1}, \overrightarrow{\mathrm{q}}_{2} ; \omega_{2}\right) \approx\left\{\begin{array}{l}
\frac{1}{q_{2}} B(3-d ; d / 2-1)(d-2)\left[\Delta\left(\omega_{2}\right)\right]^{(d-1) / 2} \text { if } \Delta\left(\omega_{2}\right)>0 \\
\frac{1}{q_{2}} B\left[\frac{1}{2},(3-d) / 2\right](d-2)\left[\Delta\left(\omega_{2}\right)\right]^{(d-1) / 2} \text { if } \Delta\left(\omega_{2}\right)<0,
\end{array}\right.
$$

where $B$ is the beta function. ${ }^{12}$ From (48), (50), and (41) one will get a nonintegrable singularity in the integrand of (17).

(c) $q_{1}>0, q_{2}=0$, and $\overrightarrow{\mathrm{q}}_{1} \cdot \overrightarrow{\mathrm{q}}_{2}=0$. This case corresponds to a contribution to the nonuniform static susceptibility. We will obtain singularities analogous to the one of $(b)$ but with $\left(q_{1}, \omega_{1}=0\right)$ replacing $\left(q_{2}, \omega_{2}\right)$ in particular in formulas (48) and (50). Putting together this result with that of (a) above, in Eq. (17), we see that the resulting singularity in $\left(q_{1}^{2}-4 k_{F}^{2}\right)^{-3 / 2}$ will occur only over an infinitesimally small region of integration over the variable $q_{2}$.

\section{Application of the case $d=2$}

We are interested in this paper in the singular behavior of $\bar{J}_{4}\left(\overrightarrow{\mathrm{q}}_{1}, \overrightarrow{\mathrm{q}}_{2} ; \omega_{2}\right)$ for $d=2$. It is readily obtained from Eqs. 
(46), (48), and (41).

(a) $q_{1}>0, q_{2}>0 \overrightarrow{\mathrm{q}}_{1} \cdot \overrightarrow{\mathrm{q}}_{2}=0 d=2$ :

$$
\operatorname{Re} \bar{J}_{4 \text { sing }}\left(\overrightarrow{\mathrm{q}}_{1}, \overrightarrow{\mathrm{q}}_{2} ; \omega_{2}\right) \approx 0 \text {. }
$$

(b) $q_{1}=0, q_{2}>0, \overrightarrow{\mathrm{q}}_{1} \cdot \overrightarrow{\mathrm{q}}_{2}=0 d=2$ :

$$
\begin{aligned}
\operatorname{Re} \bar{J}_{4 \text { sing }}\left(0, \overrightarrow{\mathrm{q}}_{2} ; \omega_{2}\right) \simeq & \Theta\left(Q_{+}^{2}-4 k_{F}^{2}\right)\left(Q_{+}^{2}-4 k_{F}^{2}\right)^{-3 / 2} \\
& +\Theta\left(Q_{-}^{2}-4 k_{F}^{2}\right)\left(Q_{-}^{2}-4 k_{F}^{2}\right)^{-3 / 2},
\end{aligned}
$$

where from Eq. (36)

$$
\begin{aligned}
& Q_{ \pm}=q_{2}\left(1 \pm \frac{2 \omega_{2}}{q_{2}}\right), \\
& {\left[\Delta\left(\omega_{2}\right)\right]_{q_{1}=0}=\frac{1}{4}\left(Q_{-}^{2}-4 k_{F}^{2}\right) .}
\end{aligned}
$$

The result in Eq. (52) deserves further comment. The dynamic susceptibility in absence of interactions, $\chi_{0}(q, \omega)$ at $d=2$, was calculated in Ref. 7 and its real part is

$$
\operatorname{Re} \chi_{0}(q, \omega)=\chi_{0}(0,0)[\Phi(\omega)+\Phi(-\omega)],
$$

where

$$
\begin{aligned}
\Phi(\omega)= & \frac{1}{2 q} Q_{+} \Theta\left(2 k_{F}-Q_{+}\right) \\
& +\Theta\left(Q_{+}-2 k_{F}\right) \frac{1}{2 q}\left[Q_{+}-\left(Q_{+}^{2}-4 k_{F}^{2}\right)^{1 / 2}\right],
\end{aligned}
$$

with $Q_{ \pm}$as given in Eq. (53) which amounts to setting $B=0$ in (7a).

By comparing Eq. (56) with Eq. (52),

$$
\operatorname{Re} \bar{J}_{\text {sing }}^{4}\left(0, \overrightarrow{\mathrm{q}}_{2} ; \omega_{2}\right) \approx \frac{\partial^{2}}{\partial \mu^{2}} \operatorname{Re} \chi_{0}\left(\overrightarrow{\mathrm{q}}_{2}, \omega_{2}\right),
$$

and this is the strongest singularity that occurs in the contribution of Fig. 3 to the calculation of the static and uniform susceptibility $\chi(q=0, \omega=0)$ at $T=0 \mathrm{~K}$, as was shown in Sec. II, Eq. (9). In particular the results of Sec. II show that these singularities are not canceled by other diagrams.

Aside from the divergence in $\left(q_{1}^{2}-4 k_{F}^{2}\right)^{-3 / 2}$ occurring in a vanishingly small region of integration over $\vec{q}_{2}$ and $\omega_{2}$ to obtain the nonuniform static susceptibility $\left(q_{1} \neq 0, \omega_{1}=0\right)$, we see from (a), (b), and (c) that the contribution of a diagram like that in Fig. 3 to $\chi\left(q_{1} \neq 0, \omega_{1}=0\right)$ will be free of nonintegrable singularities (in $\overrightarrow{\mathrm{q}}_{2}, \omega_{2}$ ), while these singularities will build up for the uniform susceptibility $\chi\left(q_{1}=0, \omega_{1}=0\right)$. The asymptotic character of the calculation does not allow us to analyze the crossover between these two behaviors.

\section{Remarks}

Another diagram entering into the lowest-order paramagnon contribution to the susceptibility is displayed in Fig. 6. It corresponds to a self-energy correction of one of the fermion lines while the contribution of Fig. 5 corresponds to a vertex correction. Of course there are two such diagrams (of the type of Fig. 6) corresponding to self-energy correction of each one of the two fermion lines. An analysis of the diagram of Fig. 6 would yield conclusions analogous to the ones obtained above concerning the diagram of Fig. 5 as is detailed in the Appendix.

Other diagrams ${ }^{13}$ also enter into the calculation of the lowest-order paramagnon contribution to the susceptibility. In any case the calculation we give in Sec. II which $a$ priori takes into account all these diagrams ensures that the singularities persist when they are all summed up.

\section{B. $2 n$-tail diagram with static external tails}

To end this section, we discuss the singularities of the $2 n$-tail ring diagram with static external tails in $d=2$ $(n=2$ in Fig. 4). These diagrams occur as static interaction potentials $U_{2 n}\left(\overrightarrow{\mathrm{q}}_{i}, \omega_{i}=0\right)$ of the $\phi^{(2 n)}$ term in the effective Landau-Ginzburg-Wilson Lagrangian for interacting paramagnons. ${ }^{5,7} \mathrm{~A}$ static $2 n$-tail diagram is given by $J_{2 n}\left(\vec{q}_{i}\right)$ as in Eq. (13), with $2 n$ fermion propagators carrying the same frequency $\epsilon$ and with external momenta $\overrightarrow{\mathrm{q}}_{1}$, $\overrightarrow{\mathrm{q}}_{2}$, and $\overrightarrow{\mathrm{q}}_{2 n-1}$ for the tails and the corresponding frequencies equal to zero. Following the same steps that led to Eq. (26) one shows that

$$
\bar{J}_{2 n}\left(\overrightarrow{\mathrm{q}}_{i}\right)=-\frac{1}{2 \pi} \frac{\partial^{2(n-1)}}{\partial \mu^{2(n-1)}} \psi\left(\overrightarrow{\mathrm{q}}_{i}\right),
$$

where $\psi\left(\vec{q}_{i}\right)$ is obtained by adding Eqs. (28a) and (28b) for $\omega_{2}=0$, and the static $\bar{J}_{2 n}\left(\overrightarrow{\mathrm{q}}_{i}\right)$ is real. For $d=2$, this gives

$$
\psi\left(\overrightarrow{\mathrm{q}}_{i}\right)=\int_{0}\left[\prod_{i} d \alpha_{i}\right] \Theta\left[1-\sum_{i} \alpha_{i}\right] \Theta(\mu+f / 2),
$$

where now the integral is over $2 n-1$ Feynman parameters $\alpha_{1}, \ldots, \alpha_{2 n-1}$, and $f\left(\vec{q}_{i}\right)$ is as given in Eq. (22) with $\omega_{2}=0$. After the change of variables in Eq. (29) with the $\bar{\alpha}_{i}$ satisfying equations analogous to (30),

$$
\overrightarrow{\mathrm{q}}_{i} \cdot \sum \bar{\alpha}_{j} \overrightarrow{\mathrm{q}}_{j}=\frac{1}{2} q_{i}^{2}, i=1, \ldots, 2 n-1
$$

one obtains

$$
\begin{aligned}
& \psi\left(\overrightarrow{\mathrm{q}}_{i}\right)=\int_{-\bar{\alpha}_{i}} \cdots \int d \beta_{1} \cdots d \beta_{2 n-1} \Theta\left(1-\sum_{i} \bar{\alpha}_{i}-\sum_{i} \beta_{i}\right) \\
& \times \Theta\left(\sum_{i, j} \beta_{i} \beta_{j} \overrightarrow{\mathrm{q}}_{i} \cdot \overrightarrow{\mathrm{q}}_{j}-\Delta\right), \\
& \Delta=\frac{1}{2} \sum_{i} \bar{\alpha}_{i} q_{i}^{2}-k_{F}^{2},
\end{aligned}
$$

from Eq. (32).

The quadratic form that occurs in the integral of Eq. (61) has coefficients that form a Gram determinant $\left|\overrightarrow{\mathrm{q}}_{i} \cdot \overrightarrow{\mathrm{q}}_{j}\right|$, then it is positive definite and it has $s$ nonzero eigenvalues $\lambda_{i}$, where $s$ is the rank of the $(2 n-1) \times(2 n-1)$ matrix $\left\|\overrightarrow{\mathrm{q}}_{i} \cdot \overrightarrow{\mathrm{q}}_{j}\right\|$. Moreover $s$ equals the number of linearly independent vectors $\overrightarrow{\mathrm{q}}_{i}$ in the set $\overrightarrow{\mathrm{q}}_{1}, \ldots, \overrightarrow{\mathrm{q}}_{2 n-1}$, hence in our case we can only have a maximum value of $s=2$ since only two vectors are linearly independent in 2D. After changing variables in Eq. (61) to diagonalize the quadratic form, and going afterwards to polar coordinates, one obtains for the singular part of 
$\psi\left(\overrightarrow{\mathrm{q}}_{i}\right)$ close to $\Delta=0$,

$\psi_{\text {sing }}\left(\overrightarrow{\mathrm{q}}_{i}\right) \approx \frac{1}{\left(\lambda_{1} \lambda_{2} \cdots \lambda_{s}\right)^{1 / 2}} \int_{0}^{\Lambda} d r r^{s-1} \Theta\left(r^{2}-\Delta\right)$,

with the result for $J^{(2 n)}\left(\overrightarrow{\mathrm{q}}_{i}\right)$ from Eq. (58),

(a) for $s=2$,

$$
J_{2 n \text { sing }}\left(\overrightarrow{\mathrm{q}}_{i}\right)=0 \text {. }
$$

(b) for $s=1$,

$$
J_{2 n \text { sing }}\left(\vec{q}_{i}\right) \approx\left\{\begin{array}{l}
0 \text { if } \Delta<0 \\
\Delta^{[1-4(n-1)] / 2} \text { if } \Delta>0 .
\end{array}\right.
$$

Equations (64) and (65) are the generalization to $2 n$ tails of Eqs. (51) and (52). For $s=1$, all momenta $\overrightarrow{\mathrm{q}}_{i}$ will be parallel, then we may write

$$
\overrightarrow{\mathrm{q}}_{j}=a_{j} \overrightarrow{\mathrm{q}}_{1}, j=2, \ldots,(2 n-1)
$$

and to satisfy Eqs. (60) implies that

$$
\sum_{j=1}^{2 n-1} \bar{\alpha}_{j} a_{j}=\frac{1}{2}, a_{j}^{2}=a_{j}, a_{1}=1,
$$

and from Eq. (62),

$$
\Delta=\frac{1}{4} q_{1}^{2}-k_{F}^{2} .
$$

It has been pointed out in Ref. 7 that paramagnon fluctuations are critical for $0 \leq q \leq 2 k_{F}$ at $d=2$; then the interaction potentials $U_{2 n}\left(\vec{q}_{i}\right)=J_{2 n}\left(\vec{q}_{i}\right)$ in a LandauGinzburg-Wilson effective Lagrangian for interacting paramagnons cannot be approximated by their value at $\overrightarrow{\mathrm{q}}_{i}=0$. If one considers the whole interval $0 \leq q_{i} \leq 2 k_{I}$ Eqs. (65) together with Eq. (68) show that $J_{2 n}\left(\vec{q}_{i}\right)$ is highly singular when $\overrightarrow{\mathrm{q}}_{1}=\overrightarrow{\mathrm{q}}_{2}=\cdots=\overrightarrow{\mathrm{q}}_{m}, m>2, q_{1}=2 k_{F}$, and $\overrightarrow{\mathrm{q}}_{m+1} \cdots \overrightarrow{\mathrm{q}}_{2 n-1}=0$, and writing an effective Lagrangian turns out to be questionable. Note, on the other hand, that when all momenta are equal to zero, $J_{2 n} \equiv 0$ for $n \geq 2$ since $J_{2 n}$ is given by the $(2 n-2)$ th derivative with respect to energy of the density of states at the Fermi level $N\left(E_{F}\right)$ with $N\left(E_{F}\right)=1 / 2 \pi$ in $2 \mathrm{D}$, i.e., is independent of the energy so that all its derivatives vanish identically. ${ }^{14}$ In such a case the quartic and higher-order terms in the Wilson Lagrangian will identically vanish and one is left with a free theory.

\section{CONCLUSIONS}

\section{A. Discussion}

In this paper we have generalized the Brovman-Kagan study of the $2 n$-multitail diagram to arbitrary dimensionality $d$ and to include the dynamics of the external tails. For a given dimension, as found by Brovman and Kagan, the degree of singularity of such diagrams increases with the number of external tails, i.e., for increasing order in the Ginzburg-Landau-Wilson series expansion. On the other hand, for a given number of external tails, e.g., the four-tail diagram which we have studied in great detail here, the degree of the singularity of such a di- agram increases with decreasing dimensionality; moreover, it depends crucially on the external momenta $\vec{q}_{i}$, $i=1,2,3$, through the rank of the $3 \times 3$ matrix $\left\|\vec{q}_{i} \cdot \overrightarrow{\mathrm{q}}_{j}\right\|$. Concerning the four-tail diagram in $2 \mathrm{D}$ with first, freestatic external legs, the results can be summarized as follows.

(1) If the momenta of the external legs are all vanishing, then the four-tail diagram and more generally the $2 n$-tail diagram with $n \geq 2$ identically vanish.

(2) If the momenta of the external legs are not all vanishing, the strongest singularity occurs when the nonvanishing momenta are all equal to $2 k_{F}$.

Concerning the four-tail diagram with two free, external legs, and the other two linked to each other, in all possible ways, as for instance, in the lowest-order paramagnon contribution to the spin susceptibility of the system (either as a vertex or a self-energy correction), the main results are the following.

(1) If the free external legs have a finite momentum $Q$ then the diagram may be singular as $\left|2 k_{F}-Q\right|^{-3 / 2}$ but only for zero momentum of the paramagnon insertion. Therefore, in the remaining integral over the inserted paramagnon momentum, the singularity will be confined to a vanishingly small volume.

(2) If the free external legs have zero momentum then the diagram is highly singular as $\left|2 k_{F}-Q^{\prime}\right|^{-3 / 2}$ where $Q^{\prime}$ is the momentum of the paramagnon insertion. Therefore, in the remaining integral over $\vec{Q}^{\prime}$ one gets a nonintegrable singularity.

An important point is that, whereas we have been able to extract and study in detail the singular part of the fourtail diagram thanks to the Brovman-Kagan method, we have been unable to calculate it in the most general case for any values of the external legs momenta, even for static legs, and neither did Brovman and Kagan. This has the following important consequences.

(1) We point out that the 2D static uniform spin susceptibility $\chi(q=0, \omega=0, T)$ cannot be computed from firstprinciples thermodynamics (in contrast to the $3 \mathrm{D}$ case) because strong singularities arise naturally in such a computation as singularities in the four-tail, and more generally, the $2 n$-tail diagram.

(2) We cannot compute the static, nonuniform susceptibility $\chi(q \neq 0, \omega=0, T)$ for arbitrary values of the momentum $q$; in particular, we are unable to conclude, even at $T=0$, whether $\chi(q, \omega=0, T=0)$, including paramagnon corrections, is maximum for a unique or a finite number of values of $q$, or if it still remains maximum for a whole continuum of values of $q$ as is the case in absence of paramagnons for the pure mean-field susceptibility

$$
\frac{\chi^{0}(q, \omega=0)}{1-I \chi^{0}(q, \omega=0)}
$$

as well as for $\chi^{0}(q, \omega=0)$ itself. This last result is most frustrating since we are left with the same puzzling question we started with: What is the nature of the magnetic transition in 2D? Is the system close to a ferromagnetic 


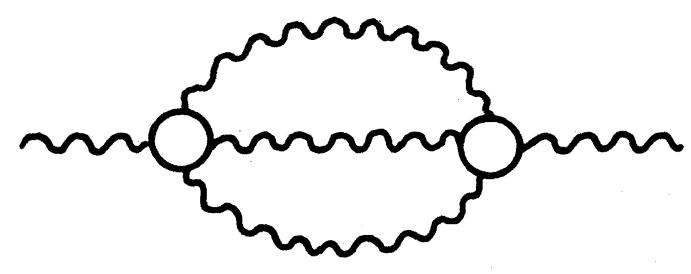

FIG. 7. Self-energy correction to the paramagnon propagator; wiggly lines are paramagnons, two closed loops are fermion lines.

instability ( $q=0$ at criticality) or to some kind of antiferromagnetic one ( $q \neq 0$ at criticality)?

In particular, we have not been able to calculate the self-energy correction to the paramagnon propagator, for instance the diagram displayed in Fig. 7, where three (instead of one in Fig. 5) fluctuation lines have to be integrated over their momenta and frequencies; such a calculation would be extremely difficult in the general case as one does not know what the critical momenta are around which an expansion could be used to render the calculation tractable. However, the calculation of such a diagram (Fig. 7) would be crucial as one of the contributions to the renormalization of the paramagnon propagator; since such a calculation cannot be performed here, one is still left with a frustrating problem: What is the renormalized paramagnon propagator in 2D?

In any case, we showed that the singularities arising in 2D prevent the use of ordinary perturbation expansion to account for fluctuation (paramagnon) effects on the susceptibility (static or dynamic one), or on any property of the $2 \mathrm{D}$ interacting fermion system as well. In particular, we confirmed what was questioned in Ref. $7(\mathrm{~b})$, i.e., one cannot assimilate to constants the various coefficients of the quartic and higher-order terms in a GinzburgLandau-Wilson expansion describing interactions among fluctuations, and that expansion itself is most likely meaningless in the light of our study. To our knowledge there has been another study by Tanaka ${ }^{15}$ of a $3 \mathrm{D}$ case where the coefficient of the quartic term in a Ginzburg-LandauWilson expansion was momentum dependent and yielded a new type of fixed point for this problem. However, the case studied by Tanaka ${ }^{15}$ was easier to handle since he chose $a$ priori a well-defined momentum dependence for the four-fluctuation interaction, i.e., for the coefficient of the quartic term in the Wilson Lagrangian. Instead, as we remarked above, we cannot provide an analytical formula for that two-fluctuation interaction but we can only study its singularities; note, in contrast, that Tanaka's interaction is perfectly well defined and not singular. Nevertheless, the point to be kept in mind is that such a momentum-dependent coefficient in the quartic term of the Wilson's expansion possibly brings in a new type of fixed point which thus may change a great deal of the critical behavior of the problem.

\section{B. Link with experiments}

As was shown above, it is not possible at present to compute, with the usual means, the spin susceptibility in the presence of fluctuations in 2D, nor to conclude what is (or what are) the critical values of $q$ or what is the nature of the magnetic instability. We also showed that all these problems arise in $2 \mathrm{D}$, from the fact that $\chi_{0}(q, \omega=0)$ is constant from $q=0$ to $q=2 k_{F}$. It can be argued that in practice and due to band structure effects, such a flatness of $\chi_{0}$ is unlikely to occur in metals. However, such a situation is perfectly relevant for $2 \mathrm{D}$ films of liquid ${ }^{3} \mathrm{He}$ in the degenerate regime. Several authors ${ }^{16}$ in the past have studied 2D Fermi liquids and have provided analytical formulas for their properties. However, all these papers ignored the flatness of $\chi_{0}(q, \omega=0)$ between $q=0$ and $q=2 k_{F}$, i.e., they all treated the problem of $2 \mathrm{D}$ Fermi liquid, implicitly assuming that, as in $3 \mathrm{D}$, only vanishing $q$ matter $\left(\xi_{\overrightarrow{\mathrm{k}}+\overrightarrow{\mathrm{q}}}-\xi_{\overrightarrow{\mathrm{k}}} \approx V_{F} q\right.$ in the Landau kinetic equation).

Under such an assumption, Ref. 16, for instance, computed that the inverse spin-diffusion coefficient $D^{-1}$ varies at low $T$ like $T^{2} \ln T$ in $2 \mathrm{D}$, while it varies like $T^{2}$ in 3D. Such a $T^{2} \ln T$ result follows straightforwardly from an integration over $q$ in $2 \mathrm{D}$, instead of $3 \mathrm{D}$, but with the same form for the dynamical response function that the one used in 3D which implies $q \rightarrow 0$ as the key value of $q$. For instance consider formula (13) of Ref. 17 for $D^{-1}$ and let us see what happens for $d=3$ and 2 if we choose to take the same form for

$$
\operatorname{Im} \chi(q, \omega, T) \sim \frac{1}{\left(1-\bar{I}+\bar{I} q^{2} / 12 k_{F}^{2}\right)^{2}} \frac{\omega}{V_{F} q} .
$$

Then at low $T$ and setting $\omega=T x$, one finds in 3D,

$$
\begin{aligned}
D^{-1} & =\frac{1}{1-\bar{I}} \frac{1}{D_{0}} \frac{1}{T} \int_{0}^{2 k_{F}} \frac{q d q}{k_{F}^{2}}\left[1-\frac{1}{4} \frac{q^{2}}{k_{F}^{2}}\right] \int_{0}^{\infty} 2 \frac{1}{\left(1-\bar{I}+\bar{I}^{2} / 12 k_{F}^{2}\right)^{2}} \frac{T^{3}}{V_{F} q} x^{2} n(x)[1+n(x)] d x \\
& \propto T^{2} \int_{0}^{2 k_{F}} \frac{d q\left(1-\frac{1}{4} q^{2} / k_{F}^{2}\right)^{2}}{\left(1-\bar{I}+\bar{I}^{2} / 12 k_{F}^{2}\right)^{2}},
\end{aligned}
$$

which is perfectly well behaved and recovers the well-known result $D^{-1} \propto T^{2}$ in 3D with a coefficient enhanced by the interactions; on the other hand, in $2 \mathrm{D}$ with the same $\operatorname{Im} \chi$ as that in $3 \mathrm{D}$, but with one less power of $q$ in integration over $q$, 


$$
\begin{aligned}
D^{-1} & =\frac{1}{1-\bar{I}} \frac{1}{D_{0}} \frac{1}{T} \int_{0}^{2 k_{F}} \frac{d q}{k_{F}}\left(1-\frac{1}{4} \frac{q^{2}}{k_{F}^{2}}\right) \int_{0}^{\infty} 2 \frac{1}{\left(1-\bar{I}+\bar{I} q^{2} / 12 k_{F}^{2}\right)^{2}} \frac{T^{3}}{V_{F} q} x^{2} n(x)[1+n(x)]^{2} d x \\
& \propto T^{2} \int_{q_{0}}^{2 k_{F}} \frac{d q\left(1-\frac{1}{4} q^{2} / k_{F}^{2}\right)}{q\left(1-\bar{I}+\bar{I} q^{2} / 12 k_{F}^{2}\right)^{2}}
\end{aligned}
$$

Here due to the divergence arising at the lower limit if $q_{0}=0$, one has to impose a lower cutoff in the $q$ integration; the natural one at very low $T$ is $q_{0}=\sqrt{T}$ and thus, one gets immediately the term $D^{-1} \propto T^{2} \ln T$ found in Ref. 16 or earlier. We insist that such a calculation is based on the assumption that altogether $\operatorname{Re} \chi_{0}(q, \omega=0)$ is maximum at $q=0$, like in $3 \mathrm{D}$, and thus $\operatorname{Im} \chi$ is proportional to $\omega / V_{F} q$, like $\operatorname{Im} \chi_{0}$ itself, although such a form ought to be revised in $2 \mathrm{D}$. Indeed, according to $\mathrm{Eq}$. (7b) (for $B=0$ ), $\operatorname{Im} \chi_{0}$ may be written as

$$
\operatorname{Im} \chi_{0} \propto \frac{\omega}{k_{F} q\left(1-q^{2} / 4 k_{F}^{2}\right)^{1 / 2}} \text { for } \omega<k_{F} q
$$

and $\omega<k_{F}^{2}\left(1-q^{2} / 4 k_{F}^{2}\right)^{1 / 2}$ together with $q<2 k_{F}$ which is already different from the 3D $\operatorname{Im} \chi_{0} \propto \omega / k_{F} q$; moreover, when $q=2 k_{F}$ strictly, Eq. (7b) shows that $\operatorname{Im} \chi_{0}$ is again different and approximately $\operatorname{sgn} \omega \sqrt{|\omega|}$, instead of $\omega$. However, as is clear from the examination of $\operatorname{Re} \chi_{0}$, the whole range $0 \leq q \leq 2 k_{F}$ is $a$ priori critical and therefore the use of $\operatorname{Im} \chi_{0} \propto \omega / k_{F} q$ in $2 \mathrm{D}$ is erroneous, and the conclusions of Ref. 16 look, to say the least, premature. Reference 16 mentions on a footnote that Ref. 7(b) of the present paper questioned the use of the Landau theory in 2D. This is not quite correct: As is well known, the Landau theory gives correct answers in critical phenomena above 4D; we recalled earlier, as proved in Ref. 5, that due to quantum effects for $T \sim 0$, the Landau or the meanfield theory gives also the correct critical exponents for 3D nearly magnetic itinerant fermions; however, as it was recalled at the beginning of the present paper and explained in Ref. 7, the structure of $\chi_{0}$ in 2D prevents any possible derivation of a Ginzburg criterion for the applicability of mean field. Therefore, the Landau theory may or may not hold in 2D paramagnon problem: in the light of the present study we show that the question is still open.

\section{ACKNOWLEDGMENTS}

One of us (A.T.) wishes to express her appreciation to Professor J. Friedel for his kind hospitality at the Laboratoire de Physique des Solides, Universite Paris-Sud, that made this collaboration possible. The other (M.T.B.-M.) wishes to acknowledge a most fruitful discussion with $\mathrm{Yu}$ Kagan who attracted her attention to Ref. 10 which plays a key role in the present study. This work is supported by the Brazilian agencies Conselho Nacional de Pesquisas (CNPq) do Brasil and Financiadora de Estudos e Projetos (FINEP).

\section{APPENDIX: LOWEST-ORDER SELF-ENERGY CONTRIBUTION TO THE SUSCEPTIBILITY}

One of the lowest-order paramagnon contributions to the susceptibility is given by the self-energy correction to one of the fermion lines of the bare bubble, as shown in Fig. 6. Here we study the singular behavior of this selfenergy correction as was done in Sec. III A for the vertex correction of Fig. 5. Note that the paramagnon propagator entering the self-energy correction of Fig. 6 has a different structure (i.e., odd number of bubbles or ladder) from the one shown in Fig. 2 (even number of bubbles) contributing to the vertex correction of Fig. 5 .

The self-energy correction to the static susceptibility then reads from Fig. 6,

$\chi_{1}^{\prime}\left(\overrightarrow{\mathrm{q}}_{1}, 0\right)=\iint \frac{d \overrightarrow{\mathrm{q}}_{3} d \omega_{3}}{(2 \pi)^{d+1}} \Pi^{\prime}\left(\overrightarrow{\mathrm{q}}_{3}-\overrightarrow{\mathrm{q}}_{1}, \omega_{3}\right) \bar{J}_{4}\left(\overrightarrow{\mathrm{q}}_{1}, \overrightarrow{\mathrm{q}}_{3} ; \omega_{3}\right)$

where $\Pi^{\prime}\left(\vec{q}_{1}, \omega\right)$ is the above-mentioned paramagnon propagator and as in Eq. (16),

$\bar{J}_{4}\left(\overrightarrow{\mathrm{q}}_{1}, \overrightarrow{\mathrm{q}}_{3} ; \omega_{3}\right)=\left[J_{4}\left(\overrightarrow{\mathrm{q}}_{1}, \overrightarrow{\mathrm{q}}_{2}, \overrightarrow{\mathrm{q}}_{3} ; \omega_{1}, \omega_{2}, \omega_{3}\right)\right]_{\overrightarrow{\mathrm{q}}_{2}=\overrightarrow{\mathrm{q}}_{1}, \omega_{1}=\omega_{2}=0}$.

Equation (A1) is the analog for Fig. 6 of Eq. (17) for Fig. 5. Following the same steps that lead to Eq. (41) we obtain

$$
\begin{aligned}
& \operatorname{Re} \bar{J}_{4}\left(\overrightarrow{\mathrm{q}}_{1}, \overrightarrow{\mathrm{q}}_{3} ; \omega_{3}\right) \\
& =-\frac{K_{d}}{(2 \pi)^{d}} \frac{\partial^{2}}{\partial \mu^{2}}\left[\psi\left(\overrightarrow{\mathrm{q}}_{1}, \overrightarrow{\mathrm{q}}_{3} ; \omega_{3}\right)+\psi\left(\overrightarrow{\mathrm{q}}_{1}, \overrightarrow{\mathrm{q}}_{3} ;-\omega_{3}\right)\right],
\end{aligned}
$$

where as in Eq. (35) for $d=2$,

$$
\psi\left(\overrightarrow{\mathrm{q}}_{1}, \overrightarrow{\mathrm{q}}_{3} ; \omega_{3}\right)=\int\left(\prod_{i} d \beta_{i} \Theta\left(\bar{\alpha}_{i}+\beta_{i}\right)\right] \Theta\left[1-\sum_{i} \bar{\alpha}_{i}-\sum_{i} \beta_{i}\right] \Theta\left(\frac{1}{2}-\bar{\alpha}_{3}-\beta_{3}\right) \Theta\left(\left[\left(\beta_{1}+\beta_{2}\right) \overrightarrow{\mathrm{q}}_{1}+\beta_{3} \overrightarrow{\mathrm{q}}_{3}\right]^{2}-\Delta\left(\omega_{3}\right)\right)
$$

for

$$
\Delta\left(\omega_{3}\right)=\frac{1}{2}\left(\bar{\alpha}_{1}+\bar{\alpha}_{2}\right) q_{1}^{2}+\frac{1}{2} \bar{\alpha}_{3}\left(q_{3}^{2}-2 \omega_{3}\right)-k_{F}^{2},
$$

and the $\alpha_{i}$ satisfying the equations

$$
\begin{aligned}
& q_{1}^{2}\left(\bar{\alpha}_{1}+\bar{\alpha}_{2}\right)+\overrightarrow{\mathrm{q}}_{1} \cdot \overrightarrow{\mathrm{q}}_{3} \bar{\alpha}_{3}=\frac{1}{2} q_{1}^{2}, \\
& \overrightarrow{\mathrm{q}}_{1} \cdot \overrightarrow{\mathrm{q}}_{3}\left(\bar{\alpha}_{1}+\bar{\alpha}_{2}\right)+q_{3}^{2} \bar{\alpha}_{3}=\frac{1}{2} q_{3}^{2}-\omega_{3} .
\end{aligned}
$$


Equations (A5) and (A6) are the analogs of Eqs. (32) and (30), respectively, for $\overrightarrow{\mathrm{q}}_{1}=\overrightarrow{\mathrm{q}}_{2}$. Note that in the present case, compatibility of the equations does not impose an extra condition equivalent to $\overrightarrow{\mathrm{q}}_{1} \cdot \overrightarrow{\mathrm{q}}_{2}=0$ in Eq. (33), which implied that the strongest singularity occurred for either $q_{1}$ or $q_{2}$ equal to zero.

Analogously to the case discussed in Sec. III B for static tails [Eq. (63)], a singularity will occur only when the rank $s$ of the $2 \times 2$ matrix $\left\|\overrightarrow{\mathrm{q}}_{i} \cdot \overrightarrow{\mathrm{q}}_{j}\right\|, i, j=1,3$ is equal to unity, implying colinearity of the momenta. We then set

$$
\overrightarrow{\mathrm{q}}_{1}=a_{1} \overrightarrow{\mathrm{q}}_{3}
$$

and we find two solutions for the set of Eq. (A6).

(i) $a_{1}=0, \bar{\alpha}_{3}=\frac{1}{2}-\omega_{3} / q_{3}^{2}$, and from Eq. (A5),

$$
\Delta\left(\omega_{3}\right)=\frac{1}{4} q_{3}^{2}\left(1-\frac{2 \omega_{3}}{q_{3}^{2}}\right)^{2}-k_{F}^{2} .
$$

From Eqs. (65), (A3), and (A4),

$$
\operatorname{Re} \bar{J}_{4 \text { sing }}\left(0 ; \overrightarrow{\mathrm{q}}_{3}, \omega_{3}\right) \approx\left\{\begin{array}{l}
{\left[\Delta\left(\omega_{3}\right)\right]^{-3 / 2} \text { if } \Delta>0} \\
0 \text { if } \Delta<0,
\end{array}\right.
$$

and by introducing Eq. (A9) in Eq. (A1) we find the same type of nonintegrable singularity that we had before for the vertex correction to the uniform static susceptibility $\left(\overrightarrow{\mathrm{q}}_{1}=0 ; \omega=0\right)$ in Eq. (52).

(ii) $a_{1}=1-2 \omega_{3} / q_{3}^{2}$, and from Eq. (A5) we obtain again in this case,

$$
\Delta\left(\omega_{3}\right)=\frac{1}{4} q_{3}^{2}\left(1-\frac{2 \omega_{3}}{q_{3}^{2}}\right)^{2}-k_{F}^{2}
$$

and the same type of singularity in $\operatorname{Re} \bar{J}_{4 \text { sing }}\left(\overrightarrow{\mathrm{q}}_{1}=a_{1} \overrightarrow{\mathrm{q}}_{3}\right.$, $\overrightarrow{\mathrm{q}}_{3} ; \omega_{3}$ ) that we had in (A9) for $q_{1}=0$. However, here $\overrightarrow{\mathrm{q}}_{1}$ and $\vec{q}_{3}$ are not independent and since one will have to integrate over $\overrightarrow{\mathbf{q}}_{3}$ and $\omega_{3}$ to get the susceptibility, the present singularity will only occur over a vanishingly small region of integration.

Therefore, we have shown as stated in the text that self-energy corrections have the same singular behavior as the vertex corrections, as expected on general grounds.
"Permanent address: Instituto di Fisica, Universidade Federal do Rio Grande do Sul, Avenida Luiz Englert, 90000 Porto Alegre, Rio Grande do Sul, Brazil.

${ }^{1}$ See, for instance, C. Herring, in Magnetism, edited by G. T. Rado and H. Shul (Academic, New York, 1966), Vol. IV.

${ }^{2}$ See, for instance, D. Pines and P. Nozières, The Theory of Quantum Liquids (Benjamin, New York, 1966).

${ }^{3}$ S. Doniach and S. Engelsberg, Phys. Rev. Lett. 17, 750 (1966); N. F. Berk and J. R. Schrieffer, ibid. 17, 433 (1966); see also, M. T. Béal-Monod, in Proceedings of the International Workshop on 3d Metallic Magnetism, Institut Laue-Langevin, Grenoble, March 1983 (in press).

${ }^{4}$ M. T. Béal-Monod, S.-k. Ma, and D. R. Fredkin, Phys. Rev. Lett. 20, 929 (1968); M. T. Béal-Monod and J. Lawrence, Phys. Rev. B 21, 5400 (1980).

${ }^{5}$ M. T. Béal-Monod and K. Maki, Phys. Rev. Lett. 34, 1461 (1975); J. Hertz, Phys. Rev. B 14, 1165 (1976).

${ }^{6}$ The value of $\bar{I}$ itself is of course modified and can be so by perturbation, but this is of minor interest since, as mentioned earlier, it is the value of $\bar{I}$ extracted from the data which is used and thus includes a priori these modifications.

7(a) M. T. Béal-Monod, Phys. Lett. 54A, 197 (1975); 59A, 157 (1976). (b) M. Gabay and M. T. Béal-Monod, Phys. Rev. B 18, 5033 (1978); M. Gabay, thesis, Orsay, 1977 (unpublished).

${ }^{8}$ See, for instance, C. Kittel, in Solid State Physics, edited by H.
Ehrenreich, F. Seitz, and A. Turnbull (Academic, New York, 1969), Vol. 22.

${ }^{9}$ K. Wilson, Phys. Rev. Lett. 2으, 548 (1972); Phys. Rev. B $\underline{4}$, 3174 (1971); 4, 3184 (1971); K. Wilson and J. Kogut, Phys. Rep. 126, 76 (1974).

${ }^{10}$ E. G. Brovman and Y. Kagan, Zh. Eksp. Teor. Fiz. 63 , 1937 (1972) [Sov. Phys._JETP 36, 1025 (1973)].

${ }^{11}$ M. T. Béal-Monod and A. Theumann, Proceedings of the International Conference on Ordering in Two Dimensions, edited by S. K. Sinha (North-Holland, Amsterdam, 1980), p. 413.

${ }^{12}$ See, for instance, I. S. Gradshteyn and I. W. Ryshik, Table of Integrals, Series, and Products (Academic, New York, 1965); M. Abranowitz and I. A. Stegun, Handbook of Mathematical Functions (Dover, New York, 1965).

${ }^{13}$ See, for instance, S.-k. Ma, M. T. Béal-Monod, and D. R. Fredkin, Phys. Rev. 174, 227 (1968), or M. T. Béal-Monod, K. Maki, and J. P. Hurault, J. Low Temp. Phys. 17, 439 (1974).

${ }^{14}$ See, for instance, p. 1091 in J. Hertz and M. A. Klenin, Phys. Rev. B 10, 1084 (1974).

${ }^{15}$ F. Tanaka, Prog. Theor. Phys. 57, 1191 (1977).

${ }^{16}$ See, for instance, K. Miyake and W. J. Mullin, Phys. Rev. Lett. 50, 197 (1983), and earlier references therein.

${ }^{17}$ See, for instance, M. T. Béal-Monod, Phys. Rev. Lett. $\underline{31,513}$ (1973). 